HEPATOBILIARY

\title{
Hepatic osteodystrophy in rats results mainly from portasystemic shunting
}

\author{
S W van der Merwe, J B van den Bogaerde, C Goosen, F F Maree, R J Milner, \\ C M Schnitzler, A Biscardi, J M Mesquita, G Engelbrecht, D Kahn, J Fevery
}

Gut 2003;52:580-585

\begin{abstract}
Background and aims: In chronic liver disease, bone disease frequently develops. The contributions of the different features of liver disease such as parenchymal inflammation, portal hypertension, and portasystemic shunting on bone metabolism have not been systematically studied. The aim of this study was to identify the features of liver disease contributing to bone disease using rat models.

Methods: Parenchymal liver disease was induced by carbon tetrachloride administration, portal hypertension by partial portal vein ligation, and portasystemic shunting by end to side anastomosis of the portal vein to the inferior vena cava. Normal and sham operated surgical animals served as controls. Serum calcium, 25-hydroxy vitamin D $(25-\mathrm{OH}$ vit D), and osteocalcin levels, and urinary deoxypyridinoline excretion were analysed. Testosterone and oestradiol levels were determined in male and female rats, respectively. Interleukin 1, interleukin 6 , and tumour necrosis factor $\alpha$ (TNF- $\alpha$ ) were determined in serum. Bone density was measured in all groups and in addition, in the surgical groups, histomorphometry was performed on undecalcified specimens of the proximal tibia. The calcium content of the femurs, removed at termination and ashed, was determined.

Results: Early parenchymal disease and portal hypertension did not affect bone metabolism or body mass. Portasystemic shunting increased bone resorption, decreased bone formation, bone density, and trabecular bone volume which were commensurate with a reduction in body mass. TNF- $\alpha$ levels were elevated and testosterone levels were low in male portasystemic shunted rats.

Conclusions: Portasystemic shunting in the rat adversely affects bone metabolism as part of a generalised catabolic state where high TNF- $\alpha$ and low testosterone and $25-\mathrm{OH}$ vit $\mathrm{D}$ levels may play a role.
\end{abstract}

Accepted for publication 28 October 2002

$\mathrm{P}$ atients with chronic parenchymal and cholestatic liver disease often have metabolic bone disorders. ${ }^{1-4}$ The pathogenesis of hepatic osteodystrophy is complex and incompletely understood. Alcohol is toxic to osteoblasts but may also affect bone indirectly through its effects on the liver. ${ }^{5}$ In autoimmune liver disease, treatment with corticosteroids promotes bone loss. Bone disease has been reported in parenchymal liver disorders independent of toxic or drug effects. ${ }^{67}$ Osteopenia is particularly common in cholestatic liver disease. ${ }^{8}$ Severe cholestasis accelerates bone loss in primary biliary cirrhosis. ${ }^{8}$ In general, the severity of bone disease correlates with the extent and duration of liver disease. Abnormal vitamin D metabolism has been reported in liver disease $^{910}$ but other studies failed to confirm this. ${ }^{11-13}$ The impact of bone disease may even extend into the post liver transplantation setting where osteopenic fractures commonly occur. $^{14}$

The aim of the present study was to determine the effects of early hepatic parenchymal disease, isolated portal hypertension, and portasystemic shunting on the development of bone disease in rat models.

\section{MATERIALS AND METHODS}

Ten week old male and female Sprague-Dawley rats were used in all experiments. Rats were housed in individual stainless steel cages in a controlled environment at a constant room temperature $\left(22^{\circ} \mathrm{C}\right)$, humidity, and 12 hour light-darkness cycles. Rats were fed standard chow (Epol, Johannesburg, South Africa) and water was given ad libitum. The approval of the ethics committee of the University of Pretoria was obtained and animals were treated according to ethical guidelines established by the University of Pretoria.

\section{Animal models and experimental design}

The experimental design consisted of two non-concurring protocols. In the first protocol, parenchymal liver disease was established and compared with a non-surgical control. In the second protocol, portal hypertension and portasystemic shunting was established and compared with a surgical control group.

Early parenchymal liver disease model: $n=10$ (males $=5$; females=5)

Liver cirrhosis in rats can be induced by weekly administration of carbon tetrachloride $\left(\mathrm{CCl}_{4}\right)$ for 16 weeks ${ }^{15}$ To induce early parenchymal disease, animals were dosed for eight weeks with $\mathrm{CCl}_{4}$ (Merck, Darmstadt, Germany). No phenobarbital was added to the drinking water because of its recognised effects on bone metabolism. ${ }^{16}$ Animals received $80 \mu \mathrm{l}$ of $\mathrm{CCl}_{4}$ at the outset with subsequent dosing based on weight loss. The intended weekly weight loss was 6-9\%. An additional five $\mathrm{CCl}_{4}$ exposed rats were sacrificed between five and 10 weeks and the livers removed for histology.

Control rats $(n=8)$ received $0.9 \%$ sodium chloride weekly by gavage.

Abbreviations: PSS, portasystemic shunting; PPVL, partial portal vein ligation; $S C$, sham operated surgical controls; $25-\mathrm{OH}$ vit $\mathrm{D}, 25$-hydroxy vitamin $\mathrm{D} ; \mathrm{CCl}_{4}$, carbon tetrachloride; u-DPD, urinary deoxypyridinoline; $B M D$, bone mineral density; $B M C$, bone mineral content; $C R P, C$ reactive protein; TNF- $\alpha$, tumour necrosis factor $\alpha$; ALT, alanine aminotransferase: ALP, alkaline phosphatase; IL, interleukin. 
Table 1 Comparison of control versus carbon tetrachloride $\left(\mathrm{CCl}_{4}\right)$ treated animals at baseline and at eight weeks, detailing liver inflammation, parameters of bone metabolism, and bone densitometry

\begin{tabular}{|c|c|c|c|c|c|}
\hline & \multicolumn{2}{|l|}{ Baseline } & \multicolumn{3}{|l|}{ Week 8} \\
\hline & Controls & $\mathrm{CCl}_{4}$ exposed animals & Controls & $\mathrm{CCl}_{4}$ exposed animals & $\mathrm{p}$ Value \\
\hline Weight (g) & $300.3(215.3-392.5)$ & $292.8(182.2-432.8)$ & $377.7(277.1-495.5)$ & $346.4(219.5-457.5)$ & NS \\
\hline AST (lu/l) & $60.22(5.12)$ & $62.93(4.35)$ & $59.22(4.80)$ & $88.75(6.74)$ & 0.02 \\
\hline ALT (lu/l) & $53.67(2.83)$ & $55.75(4.83)$ & $58.67(6.10)$ & $101.1(24.01)$ & $<0.01$ \\
\hline $\mathrm{S}-\mathrm{Ca}^{2+}(\mathrm{mmol} / \mathrm{l})$ & $2.63(0.05)$ & $2.62(0.13)$ & $2.58(0.08)$ & $2.65(0.12)$ & NS \\
\hline u-DPD ( $\mathrm{nM} / \mathrm{mM}$ creatinine) & $127.1(18.58)$ & $132.1(13.48)$ & 48.91 (13.37) & $54.99(11.24)$ & NS \\
\hline Osteocalcin $(\mathrm{pg} / \mathrm{ml})$ & $128.0(11.86)$ & $127.8(19.31)$ & $60.53(3.30)$ & $71.78(11.16)$ & NS \\
\hline $25-\mathrm{OH}$ vit $\mathrm{D}(\mathrm{ng} / \mathrm{ml})$ & $25.70(2.69)$ & $26.26(1.81)$ & 29.33 (2.89) & $20.52(1.72)$ & $<0.01$ \\
\hline \multicolumn{6}{|c|}{ High resolution $\mathrm{BMD}\left(\mathrm{g} / \mathrm{cm}^{2}\right)$} \\
\hline Proximal femur & $0.3065(0.014)$ & $0.3044(0.031)$ & $0.3793(0.044)$ & $0.3786(0.018)$ & NS \\
\hline Proximal tibia & $0.1726(0.01)$ & $0.1721(0.016)$ & $0.1982(0.021)$ & $0.2005(0.013)$ & NS \\
\hline Whole body & $0.2674(0.025)$ & $0.2654(0.037)$ & 0.3311 (0.019) & $0.3430(0.021)$ & NS \\
\hline
\end{tabular}

AST, aspartate aminotransferase; ALT, alanine aminotransferase; $\mathrm{S}-\mathrm{Ca}^{2+}$, serum calcium; $25-\mathrm{OH}$ vit $\mathrm{D}, 25$-hydroxy vitamin $\mathrm{D}$; u-DPD, urinary deoxypyridinoline; BMD, bone mineral density.

There were no significant differences between control and $\mathrm{CCl}_{4}$ treated animals at baseline.

Presinusoidal portal hypertension model (PPVL): $n=9$ (males=5; females=4)

Portal hypertension was induced by reduction of the diameter of the portal vein. ${ }^{17}$ A midline laparotomy was performed and a blunt 21 gauge needle was placed alongside the portal vein. A 3-0 silk ligature was placed around both needle and portal vein and the needle was carefully removed. Portal hypertension was confirmed at termination by portal vein pressure measurements in all PPVL animals.

\section{Portasystemic shunt model (PSS): $\mathrm{n}=10$ (males=5;}

females $=5$ )

A laparotomy was performed, the portal vein, inferior vena cava identified, and the pyloric vein ligated. The adventitia of the inferior vena cava was carefully removed. The portal vein was ligated at the hilum of the liver, transected, and the distal limb anastomosed end to side to the inferior vena cava. ${ }^{18}$ The patency of the shunt was confirmed histologically in all animals at termination.

Sham operated surgical controls $(\mathrm{SC}): \mathrm{n}=10$ (males $=5$; females=5)

A laparotomy was performed at baseline. Portal pressure measurements were conducted at termination.

The surgical groups were terminated after 16 weeks. At termination, the liver, femur, and tibia of all animals were carefully removed. The bones were carefully stripped of all soft tissue.

\section{Analytical methods in sera and urine}

Blood samples were obtained at baseline and at week 8 by puncture of the right jugular vein with a 22 gauge needle, as well as at termination in the surgical groups. Urine was collected from rats individually housed in metabolic cages following an adaptation period of 24 hours. Serum and urine samples were frozen at $-70^{\circ} \mathrm{C}$. Calcium was measured and routine liver tests and $\mathrm{C}$ reactive protein (CRP) were performed using a Perkin-Elmer 3030 atomic adsorption spectrophotometer and a Beckman CX-7 autoanalyser, respectively. Calcium levels were corrected for serum albumin in all samples. Testosterone and oestradiol levels were analysed using Access auto-analyser (Coulter Beckman, Fillerton, California, USA). 25-Hydroxy vitamin D (25- OH vit D) levels were assessed using a ${ }^{125} \mathrm{I}$-radioimmunoassay kit (DiaSorin, Stillwater, USA). Osteocalcin levels were determined according to an inhouse method developed in Leuven. ${ }^{19}$ Cytokine levels were analysed using an ELISA kit (Biotrac; Amersham Pharmacia Biotech, Buckinghamshire, UK). Urinary deoxypyridinoline crosslinks were assessed using a competitive ELISA kit (Metra Biosystems, Mountain View, USA).

\section{Bone densitometry}

Rats were anaesthetised and bone densitometry performed by dual energy $x$ ray absorptiometry using a DXA Hologic QDR 4500 (Hologic, Inc, Waltham, Massachusetts, USA). The stability of the measurement was controlled daily by scanning a phantom. Whole body and high resolution scans of the femur were carried out in $\mathrm{CCl}_{4}$ exposed animals and their controls at baseline and at week 8. Bone densitometry was performed in the surgical groups on whole body and high resolution scans of the femur at baseline, and on individual tibiae and fibulae removed at termination. The tibiae and fibulae were placed on a non- opaque polyester resin block and high resolution bone densitometry was performed using appropriate software for small animals (Hologic, Inc). Tibial measurements were used for analysis.

\section{Histomorphometry}

Individual tibiae removed at termination in the surgical groups were processed undecalcified, embedded in methylmethacrylate, and cut at $7 \mu \mathrm{m}$ thickness on a Jung $\mathrm{K}$ heavy duty microtome. Sections were stained with Masson-Goldner trichrome. Trabecular bone in the proximal $4 \mathrm{~mm}$ of the tibial metaphysis was examined for bone volume and static bone turnover variables by routine histomorphometry, using the point and intersect count method with the aid of a 100 point Zeiss eyepiece (Integrations Platte II, Zeiss, Germany) at a magnification of $\times 100$. The variables and units used are those approved by the American Society for Bone and Mineral Research..$^{20}$

\section{Portal vein pressure measurements}

Portal pressure measurements were performed at termination in the SC and PPVL animals under isofluorane 2\% anaesthesia. In control rats, portal pressures were measured by insertion of a fluid filled 20 gauge needle directly into the portal vein and in PPVL animals by inserting the needle into the distal superior mesenteric vein. The needle was connected to a transducer (Medex Medical Instrumentation, UK) calibrated for venous pressure. The external zero reference point was placed at the level of the atria. The pressure was recorded as soon as a stable reading with respiratory variations was observed

\section{Liver histology}

Liver sections were stained with haematoxylin and eosin and Masson's trichrome.

\section{Analytical methods of bone calcium content}

Rat femurs were ashed for eight hours in a muffle furnace at $600^{\circ} \mathrm{C}$, then weighed, and measured using a flexible image processing system (FIPS, CSIR, South Africa). The femurs 


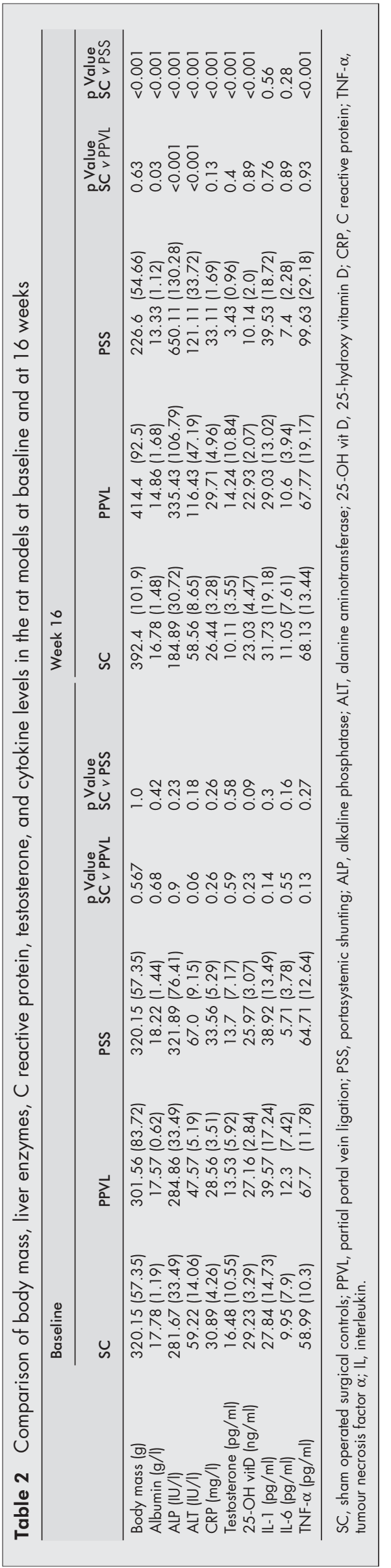

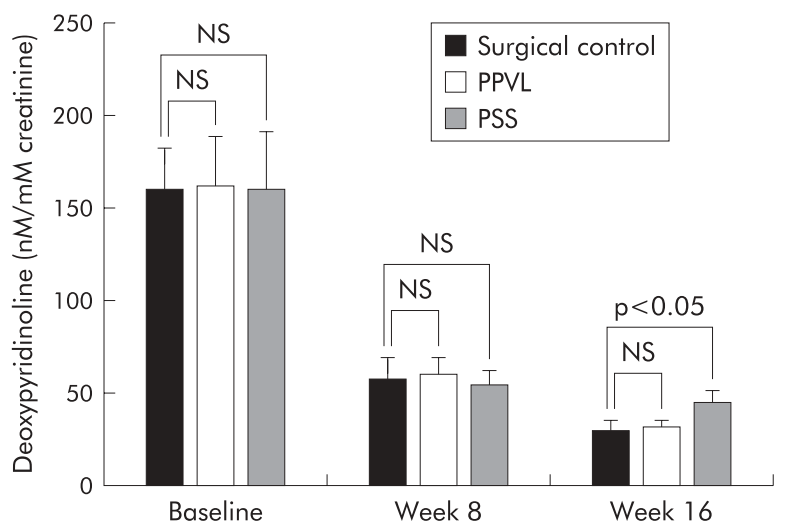

Figure 1 Urinary deoxypyridinoline levels in the rat surgical models (surgical controls, portasystemic shunt (PSS) model, and partial portal vein ligation (PPVL) model) at baseline, and at week 8 and week 16. Values are given as means (SD).

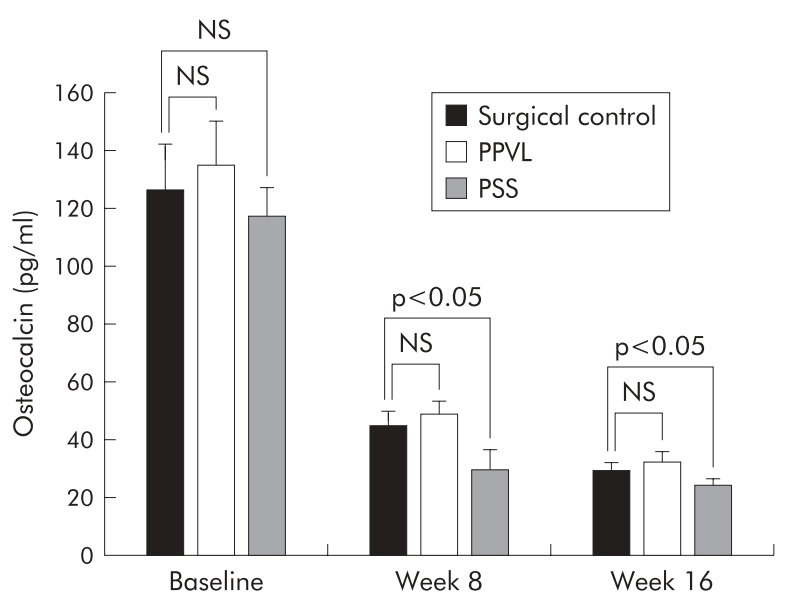

Figure 2 Serum osteocalcin levels in the rat surgical models (surgical controls, portasystemic shunt (PSS) model, and partial portal vein ligation (PPVL) model) at baseline, and at week 8 and week 16 . Values are given as means (SD).

were then dissolved in $2 \mathrm{ml}$ of $6 \mathrm{~N} \mathrm{HCl}$ and diluted $400 \times$ with demineralised/deionised water. Calcium content was determined using a Perkin-Elmer 3030 atomic absorption spectrophotometer.

\section{Statistical analysis}

Data were analysed using SigmaStat for Windows version 2.0 and SigmaPlot for Windows version 4.0. Data were checked for normality and equal variance; if passed, one way analysis of variance (ANOVA) was performed and where it failed, analysis of variance on ranks (Kruskal-Wallis) was conducted. Results are presented as means (SD), medians, or range where appropriate. Results were considered significant at $\mathrm{p}<0.05$.

\section{RESULTS}

Early parenchymal liver disease model

$\mathrm{CCl}_{4}$ exposure led to a significant increase in aspartate aminotransaminase and alanine aminotransferase (ALT) levels (table 1). At histology, characteristic changes associated with $\mathrm{CCl}_{4}$ exposure, consisting of inflammatory cell infiltrates, necrosis, and fatty changes, were observed, as has been reported previously by others. ${ }^{15}$ Except for $25-\mathrm{OH}$ vit D levels, no statistically significant differences between control and $\mathrm{CCl}_{4}$ exposed rats were observed at eight weeks in any of the variables studied relating to bone metabolism, including bone density. 25-OH vit D levels at eight weeks were significantly lower (table 1) in the treated animals compared with controls. 


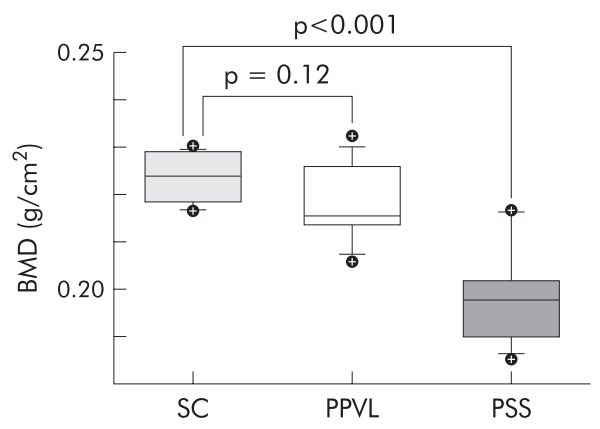

Figure 3 Tibial bone mineral density (BMD) in sham operated surgical control (SC), partial portal vein ligation (PPVL), and portasystemic shunt (PSS) rats at termination at 16 weeks. Boxes are 25th and 75 th percentiles; horizontal lines within boxes are 50th percentiles; vertical lines below and above the boxes are 5th and 95th percentiles; symbols indicate values outside the 5th and 95th percentiles.

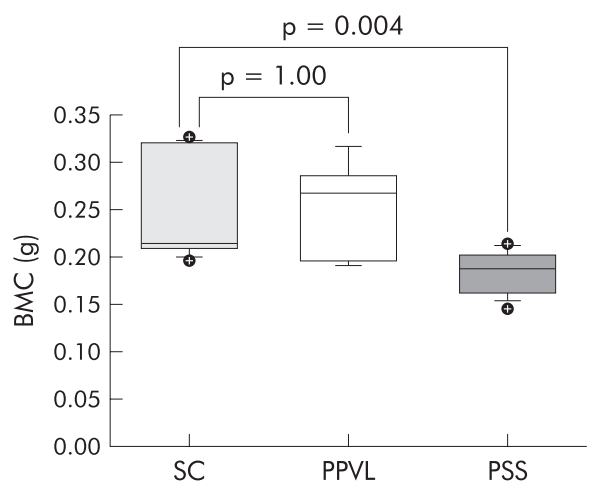

Figure 4 Tibial bone mineral content (BMC) in sham operated surgical control (SC), partial portal vein ligation (PPVL), and portasystemic shunt (PSS) rats at termination at 16 weeks. Boxes are 25th and 75th percentiles; horizontal lines within boxes are 50th percentiles; vertical lines below and above the boxes are 5th and 95th percentiles; symbols indicate values outside the 5th and 95th percentiles.

\section{Surgical series}

Similar to previously reports, ${ }^{21} 22$ liver histology showed varying degrees of atrophy in acinar zone 3 in both PPVL and PSS groups, although atrophy was more pronounced in PSS animals. Mild fatty change was only observed in the PSS group. Portal pressures were significantly elevated in PPVL animals compared with SC (19.8 (6.25) v 6.8 (2.49) $\mathrm{mm} \mathrm{Hg}$; $\mathrm{p}=0.002$ ).

The results of biochemical and other parameters studied are presented in tables 1 and 2. Body mass was significantly lower in PSS animals at 16 weeks, as has been reported previously. Serum calcium concentrations, corrected for serum albumin, did not differ between the groups at termination. ALT levels were elevated in both PPVL and PSS groups at termination. As shown in table 2, albumin, testosterone, and $25-\mathrm{OH}$ vit $\mathrm{D}$ levels were significantly decreased, and tumour necrosis factor $\alpha$ (TNF- $\alpha$ ) and CRP levels significantly increased only in the PSS group at 16 weeks. Alkaline phosphatase (ALP) levels were significantly elevated in PSS and PPVL rats at termination. Markedly raised ALP levels in the PSS group may reflect both liver and bone specific isoenzymes. Oestradiol levels were assessed in female rats but were highly variable ranging between undetectable to more than $2000 \mathrm{pg} / \mathrm{ml}$, precluding interpretation of this parameter. Interleukin (IL)-land IL-6 levels did not differ at termination between the groups.

Bone resorption, as assessed by urinary deoxypyridinoline (u-DPD) excretion, is shown in fig l. A decrease in u-DPD in all groups from baseline to eight and 16 weeks is seen and may represent an aging phenomenon. Despite this overall decrease, u-DPD excretion was significantly higher in PSS rats at 16 weeks, indicating greater bone resorption. Bone formation, as assessed by serum osteocalcin levels, is shown in fig 2. Osteocalcin had decreased in all groups by weeks 8 and 16, again indicating an age related change. Osteocalcin levels were significantly lower in PSS animals at eight and 16 weeks compared with SC, indicating a decrease in bone formation. Similar to early parenchymal disease, $25-\mathrm{OH}$ vit D was significantly lower in shunted rats compared with controls at 16 weeks (table 2 ).

BMD performed at baseline using high resolution scans of the femur and whole body measurements did not differ between groups. In contrast, BMD performed on individual tibiae at termination showed lower bone density in PSS animals compared with PPVL rats and SC (figs 3, 4). Tibial BMC was also significantly lower in PSS animals at 16 weeks. Tibial BMD did not differ between male and female rats in the PSS group $(\mathrm{p}=0.756)$, excluding possible sex differences.

Histomorphometric analysis at 16 weeks revealed that shunted rats had significantly lower tibial trabecular bone volume, osteoid volume, and osteoid surface compared with controls (table 3, fig 5). Osteoid seams appeared thin. Resorption surface was significantly greater in PSS rats than in controls. These parameters were not different between SC and PPVL rats at 16 weeks.

Table 4 summarises the results of bone measurements and calcium content. Femur length and mass were lower in shunted animals than in controls but when corrected for body mass these differences changed in that femur mass per $100 \mathrm{~g}$ body mass in PSS rats was significantly greater than in SC. Bone calcium estimation showed that only the PSS group had less calcium per femur and less calcium per mm of bone compared with controls, but calcium per mg of bone did not differ between groups. This absence of an excessive osteoid seam thickness rules out a mineralisation defect. The calcium content per femur in the PSS group was lower than in controls because the bones were smaller, and the calcium content per $\mathrm{mm}$ of bone was lower because femoral thickness was less in these animals.

\section{DISCUSSION}

Osteopenia has been previously reported in rats made cirrhotic by $\mathrm{CCl}_{4}$ administration. ${ }^{16}$ However, as in humans, it remains unclear which aspect of cirrhosis is responsible for bone loss. The present study demonstrated that experimental portasystemic shunting had the most deleterious effect on bone.

Complete portasystemic shunting led to significantly lower bone formation, greater bone resorption, lower trabecular bone volume, and smaller bone size compared with controls. The lower tibial trabecular bone volume was reflected in lower bone density, and both lower trabecular bone volume and smaller bone size in lower BMC in PSS rats. The smaller bone size is interpreted as an expression of an overall failure to thrive, and of stunted growth. Indeed, the reduction in bone mass together with body mass suggests that bone loss occurs as part of a generalised catabolic state. The presumed partial shunting induced by collaterals in the portal vein ligation model did not have the same adverse effect on body mass, growth, or bone, nor did early parenchymal disease.

Suppression of bone formation in the PSS group was expressed by lower values for osteocalcin, osteoid surface, and osteoid volume, and greater bone resorption by higher values for u-DPD and increased resorption surfaces observed in PSS animals but not in controls. This picture suggests uncoupling of bone formation from resorption in favour of resorption. Contributions to these bone abnormalities by failure to thrive, stunted growth, and malnutrition cannot be ruled out. 
Table 3 Comparison of histomorphometric parameters: trabecular bone volume, osteoid volume, and osteoid surface, and resorption surface in the proximal tibia of rats at 16 weeks

\begin{tabular}{lccccc}
\hline & SC & PPVL & PSS & $\begin{array}{c}\text { p Value } \\
\text { SC } v \text { PPVL }\end{array}$ & $\begin{array}{l}\text { p Value } \\
\text { SC } v \text { PSS }\end{array}$ \\
\hline Trabecular bone volume (\%) & $13.2(1.35)$ & $14.17(2.23)$ & $7.92(0.99)$ & 0.36 & $<0.001$ \\
Osteoid volume (\%) & $7.16(1.23)$ & $5.17(1.47)$ & $4.74(0.74)$ & 0.051 & 0.001 \\
Osteoid surface (\%) & $9.71(1.04)$ & $11.33(2.34)$ & $6.06(1.33)$ & 0.234 & $<0.001$ \\
Resorption surface (\%) & $0.94(0.07)$ & $1.73(0.59)$ & $3.38(0.96)$ & 0.061 & $<0.001$ \\
\hline \multicolumn{7}{l}{ SC, sham operated surgical controls; PPVL, partial portal vein ligation; PSS, portasystemic shunting. } \\
\end{tabular}

A

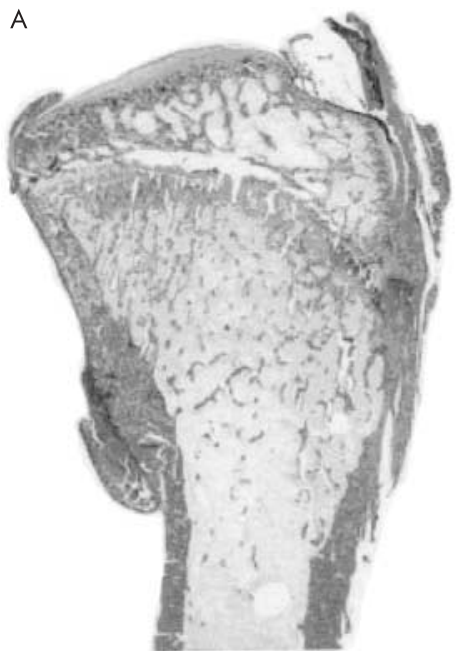

B

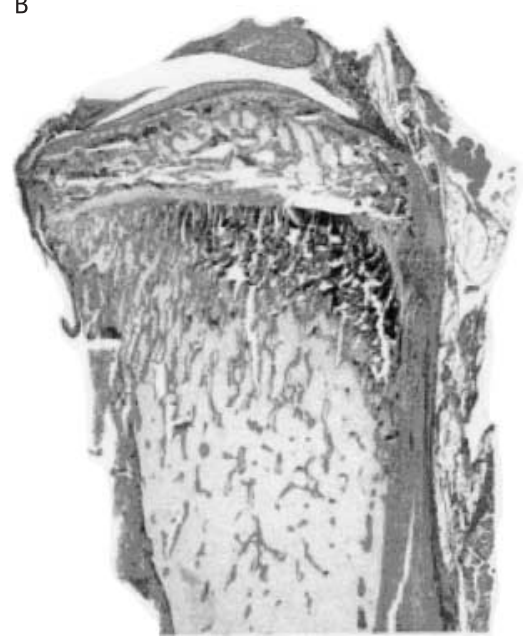

C

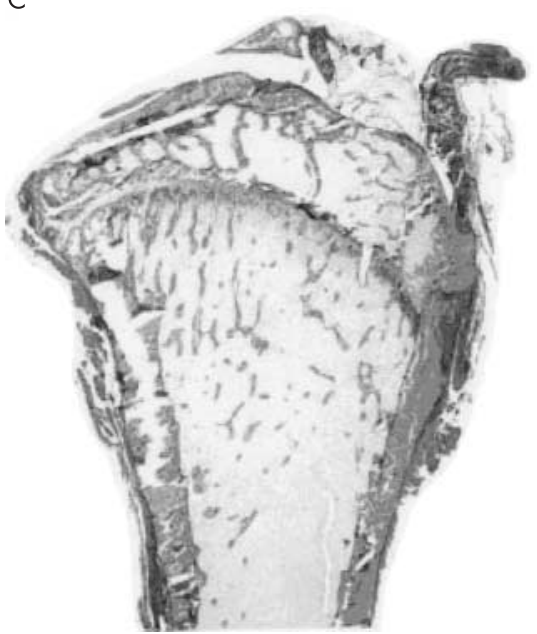

Figure 5 Photomicrograph of undecalcified proximal tibia from (A) sham operated surgical control (SC), (B) partial portal vein ligation (PPVL) rat, and (C) portasystemic shunt (PSS) rat. The PSS rat had the lowest trabecular bone volume. Masson-Goldner trichrome stain; original magnification $\times 8$.

Table 4 Comparison of femur dimensions, composition, and tibial bone mineral density in rats at termination at 16 weeks

\begin{tabular}{|c|c|c|c|c|c|}
\hline & $S C(n=10)$ & $\operatorname{PPVL}(n=9)$ & PSS $(n=10)$ & $\begin{array}{l}\text { p Value } \\
\text { SC } v \text { PPVL }\end{array}$ & $\begin{array}{l}\mathrm{p} \text { Value } \\
\text { SC } \vee \text { PSS }\end{array}$ \\
\hline Femur length $(\mathrm{mm})$ & $30.47 \quad(2.36)$ & $30.78 \quad(1.94)$ & $27.83 \quad(1.25)$ & 0.86 & 0.008 \\
\hline Mid shaft thickness (mm) & $4.13(0.46)$ & $3.98 \quad(0.31)$ & $3.63(0.30)$ & 0.44 & 0.017 \\
\hline Femur mass (g) & $0.416(0.09)$ & $0.385(0.06)$ & 0.295 (0.03) & 0.37 & $<0.001$ \\
\hline $\mathrm{mg} \mathrm{Ca}{ }^{2+} / \mathrm{femur}$ & $70.92(14.52)$ & $66.29(14.62)$ & $50.69 \quad(5.40)$ & 0.50 & 0.003 \\
\hline Femur mass $/ 100 \mathrm{~g}$ body mass & $0.108(0.009)$ & $0.100(0.02)$ & $0.137(0.03)$ & 0.08 & 0.01 \\
\hline $\mathrm{mg} \mathrm{Ca}^{2+} / \mathrm{mm}$ bone & $2.31(0.30)$ & $2.18 \quad(0.36)$ & $1.82(0.13)$ & 0.40 & 0.002 \\
\hline $\mathrm{mg} \mathrm{Ca}{ }^{2+} / \mathrm{mg}$ bone & $0.164(0.03)$ & $0.172(0.02)$ & $0.172(0.003)$ & 0.21 & 0.34 \\
\hline Tibial BMD $\left(\mathrm{g} / \mathrm{cm}^{2}\right)$ & $0.223(0.005)$ & $0.218(0.009)$ & $0.199(0.01)$ & 0.12 & $<0.001$ \\
\hline Tibial BMC (g) & $0.244(0.05)$ & $0.253(0.05)$ & $0.184(0.02)$ & 1.00 & 0.004 \\
\hline
\end{tabular}

The discrepancy between the rise in femur mass per $100 \mathrm{~g}$ of body weight and the decline in trabecular bone volume in the PSS group may be due to the fact that the femur consists predominantly of cortical bone whereas histomorphometry is routinely carried out on trabecular bone. Trabecular bone loss is known to occur earlier and more rapidly than cortical bone loss because of the larger surface area available for resorption. The duration of the experiment may have been insufficient for cortical bone loss to become apparent. The rise in femur mass per $100 \mathrm{~g}$ of body mass in PSS rats at 16 weeks may be explained on similar grounds: body fat and lean body mass are presumably lost earlier and more rapidly than cortical bone mass in the femur so that the lag in cortical bone loss gives rise to an apparent increase in bone mass relative to body mass. ${ }^{23}$
Whether the findings in the rat can be applied to adult humans remains unclear: rat bone continues to grow throughout life and does not exhibit remodelling. A similar study in a larger animal that has both closed physes in adulthood and bone remodelling may be more applicable to human bone pathology.

The cytokines IL-1, IL- 6 , and TNF- $\alpha$ have been shown to influence different aspects of bone metabolism. ${ }^{24-28}$ IL-1, IL-6, and TNF- $\alpha$ are known to be elevated in liver disease. ${ }^{24}$ In our study, TNF- $\alpha$ levels were increased in PSS animals at termination and may have contributed to the development of osteopenia.

Chronic liver disease, especially in males, can result in gonadal dysfunction with low testicular mass and decreased 
estosterone levels. ${ }^{17}$ Interestingly, portasystemic shunting, but ot portal hypertension, has been reported to result in gonadal injury in a rat model. ${ }^{29}$ Low testosterone levels were confirmed in the male PSS rats in our study and may have contributed to the development of osteopenia. Despite similar changes in bone parameters, oestradiol levels did not seem to have the same influence in female rats

The role of vitamin D in hepatic osteodystrophy remains unclear. Vitamin D levels were decreased in the PSS and early parenchymal liver disease groups but despite this, bone mineralisation remained unaffected. Impaired bone formation in chronic liver disease may be responsible for failure of osteomalacia to become apparent as mineralisation defects develop only in newly laid down osteoid. Earlier studies suggested that a decrease in 25-hydroxylase activity was responsible for the osteodystrophy found in bile duct ligated rats ${ }^{30}$ This could not be confirmed in $\mathrm{CCl}_{4}$ treated rats. ${ }^{31}$ Vitamin $\mathrm{D}$ metabolism has not been studied in portacaval shunting. $25-\mathrm{OH}$ vitamin D levels are normal in primary biliary cirrhosis except in the terminal phases when osteoporosis is established..$^{32}$ In addition, no association was found between vitamin $\mathrm{D}$ receptor gene polymorphisms and bone mass in primary biliary cirrhosis. ${ }^{8}$

In summary, this study demonstrated that of the three features found in chronic liver disease, namely parenchymal inflammation, portal hypertension, and portasystemic shunting, shunting had the most pronounced effect on bone as part of a generalised catabolic state. Although the precise mechanism whereby bone disease is induced remains unclear, cytokine activation, low sex hormone and vitamin D levels, or a combination of these factors may play a role.

\section{ACKNOWLEDGEMENTS}

The authors acknowledge the contributions of Professor Ian Simpson Department of Pathology, University of Pretoria, for liver histology, and Professor Roger Bouillon, Department of Endocrinology, Leuven, Belgium, for determination of osteocalcin levels, as well as the excellent secretarial assistance of Ms Gezina Kies. This study was supported by the Hepatology Research fund, ASN 5991, University of Pretoria.

\section{Authors' affiliations}

S W van der Merwe, J B van den Bogaerde, C Goosen, F F Maree, R J Milner, Department of Internal Medicine and Gastroenterology, University of Pretoria, South Africa

C M Schnitzler, A Biscardi, J M Mesquita, Department of Orthopaedic Surgery and MRC Mineral Metabolism Research Unit, University of the Witwatersrand, Johannesburg, South Africa

G Engelbrecht, D Kahn, Department of Surgery, Grootte Schuur Hospital, University of Cape Town, South Africa

J Fevery, Departments of Hepatology and Internal Medicine, Catholic University of Leuven, Belgium

\section{REFERENCES}

1 Diamond T, Stiel D, Lunzer $M$, et al. Osteoporosis and skeletal fractures in chronic liver disease. Gut 1990;31:82-7.

2 Chen CC, Wang SS, Jeng FS, et al. Metabolic bone disease of liver cirrhosis: is it parallel to the clinical severity of cirrhosis? J Gastroenterol Hepatol 1996;11:417-21.

3 Bonkovsky HL, Hawkins M, Steinberg K, et al. Prevalence and prediction of osteopenia in chronic liver disease. Hepatology 1990; 12:273-80.

4 Tsuneoka K, Tameda Y, Takase K, et al. Osteodystrophy in patients with chronic hepatitis and liver cirrhosis. J Gastroenterol 1996;31:669-78.

5 Peris P, Pares A, Guanabens N, et al. Reduced spinal and femoral bone mass and deranged bone mineral metabolism in chronic alcoholics. Alcohol Alcohol 1992;27:619-25.
6 Idilman R, De Maria N, Uzunalimoglu O, et al. Hepatic osteodystrophy: a review. Hepatogastroenterology 1997:44:574-81.

7 Gallego-Rojo FJ, Gonzalez-Calvin JL, Munoz-Torres M, et al. Bone mineral density, serum insulin-like growth factor $I$, and bone turnover markers in viral cirrhosis. Hepatology 1998;28:695-9.

8 Pares A, Guanabens N, Alvarez L, et al. Collagen type $|\alpha|$ and vitamin $D$ receptor gene polymorphisms and bone mass in primary biliary cirrhosis. Hepatology 2001;33:554-60.

9 Davies M, Mawer EB, Klass HJ, et al. Vitamin D deficiency, osteomalacia and primary biliary cirrhosis. Response to orally administered vitamin D3. Dig Dis Sci 1983;28:145-53.

10 Dibble JB, Sheridan P, Hampshire R, et al. Osteomalacia, vitamin D deficiency and cholestasis in chronic liver disease. QJ Med 1982;51:89-103

11 Floreani A, Zappala F, Fries W, et al. A 3-year pilot study with 1,25dihydroxyvitamin $D$, calcium, and calcitonin for severe osteodystrophy in primary biliary cirrhosis. J Clin Gastroenterol 1997:24:239-44.

12 Herlong HF, Recker RR, Maddrey WC. Bone disease in primary biliary cirrhosis: histologic features and response to 25 -hydroxy-vitamin D. Gastroenterology 1982;83: 103-8.

13 Matloff DS, Kaplan MM, Neer RM, et al. Osteoporosis in primary biliary cirrhosis: effects of 25-hydroxyvitamin D3 treatment. Gastroenterology 1982:83:97-102.

14 Hay JE, Malinchoc M, Dickson ER. A controlled trial of calcitonin therapy for the prevention of post-liver transplantation atraumatic fractures in patients with primary billiary cirrhosis and primary sclerosing cholangitis. $J$ Hepatol 2001;34:292-8.

15 Proctor E, Chatamra K. High yield micronodular cirrhosis in the rat. Gastroenterology 1982;83:1 183-90.

16 Cemborain A, Castilla-Cortazar I, Garcia M, et al. Osteopenia in rats with liver cirrhosis: beneficial effects of IGF-I treatment. J Hepatol 1998;28:122-31.

17 Van Thiel DH, Gavaler JS, Slone FL, et al. Is feminization in alcoholic men due in part to portal hypertension: A rat model. Gastroenterology 1980;78:81-91.

18 Robson SC, Jaskiewicz K, Engelbrecht G, et al. Haemostatic and immunological sequelae of portacaval shunt in rats. Liver 1995; 15:293-9.

19 Verhaeghe J, Van Herck E, Van Bree R, et al. Osteocalcin during the reproductive cycle in normal and diabetic rats. J Endocrinol 1989; 120:142-51.

20 Parfitt AM, Drezner MK, Glorieux FH, et al. Bone histomorphometry: standardization of nomenclature, symbols and units. J Bone Miner Res 1987; 2:595-610.

21 Agnihotri N, Bhusnurmath SR, Narasimhan KL, et al. Experimental extrahepatic obstruction of portal vein: documentation of histopathological alterations in liver and extrahepatic tissues. $J$ Gastroentrol Hepatol 1996;11:971-7.

22 Benjamin IS, Ryan CJ, Engelbrecht GH, et al. Portacaval transposition in the rat: definition of a valuable model for hepatic research. Hepatology 1984:4:704-8.

23 Picardi A, De Oliveira AC, Muguerza B, et al. Low doses of insulin-like growth factor-l improve nitrogen retention and food efficiency in rats with early cirrhosis. J Hepatol 1997;26:191-202.

24 Kimble RB. Alcohol, cytokines, and estrogen in the control of bone remodeling. Alcohol Clin Exp Res 1997:21:385-91.

25 Pfeilschifter J, Chenu C, Bird A, et al. Interleukin- 1 and tumor necrosis factor stimulate the formation of human osteoclast-like cells in vitro. J Bone Miner Res 1989;4:113-18

26 Gowen M, Mundy GR. Actions of recombinant interleukin 1, interleukin 2 and interferon-gamma on bone resorption in vitro. J Immunol $1986 ; 136: 2478-82$.

27 De La Mata, J, Uy HL, Guise TA, et al. Interleukin-6 enhances hypercalcemia and bone resorption mediated by parathyroid hormone-related protein in vivo. J Clin Invest 1995;95:2846-52.

28 Thomson BM, Mundy GR, Chambers TJ. Tumor necrosis factors alpha and beta induce osteoblastic cells to stimulate osteoclastic bone resorption. J Immunol 1987; 138:775-9.

29 Van Thiel DH, Gavaler JS, Cobb CF, et al. An evaluation of the respective roles of portosystemic shunting and portal hypertension in rats upon the production of gonadal dysfunction in cirrhosis. Gastroenterology 1983;85:154-9.

30 Bolt MJ, Sitrin MD, Favus M, et al. Hepatic vitamin D 25-hydroxylase: inhibition by bile duct ligation or bile salts. Hepatology 1981;1:436-40.

31 Nakano A, Kanda T, Abe $\mathrm{H}$. Bone changes and mineral metabolism disorders in rats with experimental liver cirrhosis. J Gastroenterol Hepatol 1996:11:1143-54.

32 Bouillon R, Auwerx J, Dekeyser L, et al. Serum vitamin D metabolites and their binding protein in patients with liver cirrhosis. J Clin Endocr Met 1984;59:86-9 